\title{
Using Dynamic Voltage Drive in a Parallel-Plate Electrostatic Actuator for Full-Gap Travel Range and Positioning
}

\author{
Luis Alexandre Rocha, Edmond Cretu, and Reinoud F. Wolffenbuttel
}

\begin{abstract}
The nonlinear dynamics of the parallel-plate electrostatically driven microstructure have been investigated with the objective of finding a dynamic voltage drive suitable for full-gap operation. Nonlinear dynamic modeling with phase-portrait presentation of both position and velocity of a realistic microstructure demonstrate that instability is avoided by a timely and sufficient reduction of the drive voltage. The simulation results are confirmed by experiments on devices fabricated in an epi-poly process. A 5.5-V peak harmonic drive voltage with frequency higher than $300 \mathrm{~Hz}$ allows repetitive microstructure motion up to $70 \%$ of gap without position feedback. The results of the analysis have been applied to the design of a new concept for positioning beyond the static pull-in limitation that does include position feedback. The measured instantaneous actuator displacement is compared with the desired displacement setting and, unlike traditional feedback, the voltage applied to the actuator is changed according to the comparison result between two values. The "low" level is below the static pull-in voltage and opposes the motion, thus bringing the structure back into a stable regime, while the "high" level is larger than the static pull-in voltage and will push the structure beyond the static pull-in displacement. Operation is limited only by the position jitter due to the time delay introduced by the readout circuits. Measurements confirm flexible operation up to a mechanical stopper positioned at $2 \mu \mathrm{m}$ of the $2.25 \mu \mathrm{m}$ wide gap with a $30 \mathrm{~nm}$ ripple.

[1519]
\end{abstract}

Index Terms-Dynamic pull-in, electrostatic actuators, nonlinear dynamics, pull-in, stable extended travel.

\section{INTRODUCTION}

$\mathbf{E}$ LECTROSTATIC actuation is not restricted to the $\mu$-domain. Already in the nineteenth century, experimental electrostatic motors were reported. A narrow gap $(<1 \mathrm{~mm})$ between precisely aligned stator and rotor plates was essential for generating sufficient electrostatic force to get the motor running. This minimum gap width was limited at a given voltage by breakdown. What makes the $\mu$-domain unique is the fact that device operation is not limited by electrostatic breakdown field, but rather by pull-in of the microstructure. This property is due to Paschen law, which indicates that the value of the breakdown field is governed by the number of ionizable molecules available and thus increases with decreasing gap width at constant air pressure. Typical values range from $3.10^{6} \mathrm{~V} / \mathrm{m}$ in a $10-\mathrm{mm}$

Manuscript received February 15, 2005; revised July 12, 2005. Subject Editor N. C. Tien.

L. A. Rocha and R. F. Wolffenbuttel are with the Delft University of Technology, Department of Microelectronics, 2628 CD Delft, The Netherlands (e-mail: L.Rocha@ewi.tudelft.nl).

E. Cretu is with the Melexis, Tessenderlo, Belgium.

Digital Object Identifier 10.1109/JMEMS.2005.859099 gap to $10^{8} \mathrm{~V} / \mathrm{m}$ in a 5- $\mu \mathrm{m}$-wide gap at ambient pressure [1], [2]. Considering the value for the narrow-gap breakdown field and the dimensions of a typical microstructure, breakdown is unlikely to take place in a microelectromechanical system (MEMS). Therefore, the electromechanical coupling that is by necessity weak at the macroscopic level can be much stronger at (sub) micron dimensions, which enables innovative MEMS applications [3]. Other benefits that come with the downscaling of the characteristic dimensions of the elements to the micrometer size are the reduced influence of effects related to the device volume, such as gravity, as compared with surface effects, such as adhesive and friction effects [4].

However, since the electrostatic force in a vertical field is inversely proportional to the square of the deflection and the restoring force of an electrostatically actuated beam is, in a first approximation, linear with deflection, an unstable system results and the suspended beam (or rotor) crashes on the stator in case of a deflection, $v$, beyond a critical value, $\nu_{\text {crit }}$. The pull-in voltage, $V_{\mathrm{pi}}$, is defined as the voltage that is required to obtain this critical deflection and is determined by the beam material, beam dimensions, residual stress and electrode dimensions [5].

This pull-in phenomenon was first reported in 1967 as a property of the resonant gate transistor (RGT) [6] and basically reflects one of the consequences of the tight and highly nonlinear electromechanical coupling. The electrostatic forces are inversely proportional to the square of the capacitor gap and also damping forces in a microscale gap are highly nonlinear. Pull-in is amongst the most studied MEMS phenomena [5]-[9] and the mechanisms involved are still not fully understood. The simplified analysis assumes a quasistatic regime, which reduces the problem to finding the equilibrium between mechanical and electrostatic forces (i.e., the damping is neglected) and results in a sudden pull-in at a well-defined pull-in voltage at a displacement of $1 / 3$ of gap for 1 degree-of-freedom (1-DOF) structures [5]. However, when the changes in the applied voltage are sufficiently fast, the quasistatic regime does not apply and the static pull-in analysis becomes invalid. The damping forces and mass inertia need to be included in the model for a meaningful study of the dynamic pull-in behavior of the structure [10]-[13].

The focus of this paper is on the dynamics of parallel-plate electrostatically driven microstructures, with the purpose of achieving stable displacements beyond the static pull-in limitation. Electrostatic parallel-plate actuation is limited to displacements up to $1 / 3$ of the gap due to pull-in. This factor limits the use of electrostatic parallel-plate actuation in many 


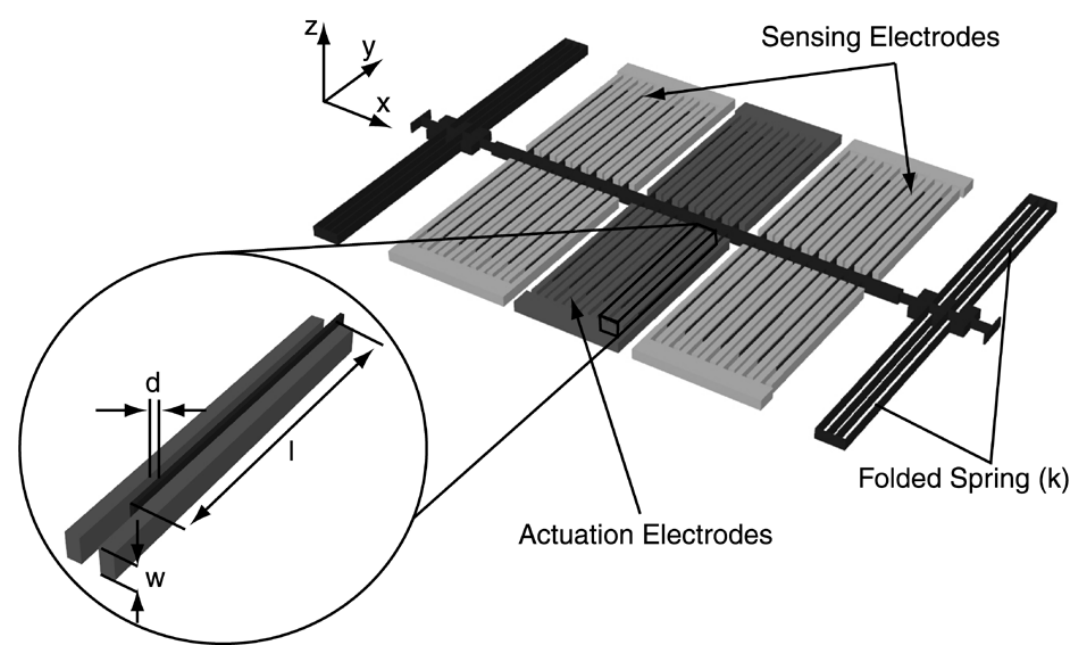

Fig. 1. Drawing of the parallel-plate electrostatic microactuator.

applications. In order to overcome this limitation some techniques have been investigated: geometry leverage [14], series feedback capacitor [15]-[17], current drive methods [18]-[20] and closed-loop voltage control [21]-[23].

Stable displacement over the full available range has not been achieved in these approaches reported, except for the geometry leverage technique [14], which is limited by the higher voltage levels required and the larger dimensions. It is interesting to note that these studies overlooked the opportunities provided by the dynamics of the devices. A fundamental MEMS dynamic characteristic, as it will be discussed here, is the achievable dynamic displacement beyond the static pull-in limitation. This is a fundamental issue and provides the means for a new closed-loop voltage control method. This new voltage drive approach provides a simple and effective way to achieve stable displacements beyond the static pull-in limitation. Both simulations and fabricated microstructures are applied to demonstrate the concept. Stable displacements along the full available gap are experimentally achieved, and the simplicity and effectiveness of the method are an added value to parallel-plate electrostatic actuators.

First the nonlinear dynamics is studied using a dynamic MEMS model. Fabricated devices are subsequently used to confirm both the modeling and the analysis and to validate the proposed voltage drive method for extending the travel range to full-gap.

\section{MEMS NONLINEAR DYNAMICS}

The nonlinearities in electrostatic actuated parallel-plate micromachined actuators are in several energy domains, which make the nonlinear problem difficult to solve. The consequences of the nonlinear nature of the problem are most profound in the dynamics of the system. Furthermore, the nonlinearity of the systems is generally poorly specified. Instead of quantitative solutions (which can be obtained only numerically in nearly all cases), qualitative aspects, such as type of solutions, stability of solutions, and bifurcation of new solutions, are often considered of greater interest [24]. In this Section quantitative and qualitative tools are used for the analysis of a nonlinear dynamic MEMS. First, a dynamic model is built which allows solving the differential equation of motion. Subsequently, a phase portrait is constructed and the qualitative aspects of the system are analyzed. This phase portrait is used as a tool for analyzing dynamic techniques to achieve stable displacements beyond the static pull-in limitation.

\section{A. Dynamic Model}

In the modeling of MEMS, four energy domains are usually considered: thermal, mechanical, fluidic and electrical. Usually, the effect of changes in temperature on dynamic performance can be neglected and the use of a static temperature is sufficient. The mechanical and electrical domains are relatively easy to model and the fluidic domain is the most demanding modeling challenge.

The micromechanical structure used for the modeling and nonlinear analysis is basically a planar movable beam with folded suspension on both ends and electrodes extending perpendicular to the axial direction. As shown in Fig. 1, one set of stator electrodes in the same plane is used for electrostatic actuation in the direction normal to the electrode area and two other set of electrodes are used for capacitive displacement measurement. Assuming no external mechanical force applied (no acceleration), the movement of such a parallel-plate electrostatic actuator is described by

$$
F_{m}+F_{b}+F_{k}=F_{\text {elec }}
$$

where $\mathrm{F}_{\mathrm{m}}$ is the mass contribution $(m \ddot{x}), \mathrm{F}_{\mathrm{b}}$ is the force caused by the damping, $\mathrm{F}_{\mathrm{k}}$ is the spring force $(k x)$ and $\mathrm{F}_{\text {elect }}$ is the electrostatic force $\left((1 / 2) C^{\prime}(x) V^{2}\right)$, where $C^{\prime}(x)$ is the partial derivative of the capacitance with respect to the displacement $x$.

1) Damping Model: For structures in which only the size of the small gap between two plates changes in time, the pressure changes $p$ relative to the wall velocity are described by the following Reynolds equation [25]:

$$
\frac{d^{3} Q_{\mathrm{pr}}}{12 \eta}\left(\frac{\partial^{2} p}{\partial x^{2}}+\frac{\partial^{2} p}{\partial y^{2}}\right)=\frac{d}{p_{a}} \frac{\partial p}{\partial t}+\frac{\partial d}{\partial t}
$$

where $p_{a}$ is the ambient pressure, $\eta$ the gas viscosity, $Q_{\mathrm{pr}}$ describes the relative flow rate coefficient and $d$ the gap between the surfaces.

An analytical solution for the forces acting on the surfaces can be found if some conditions are assumed [26]: the moving plate 
is rigid and moves in the direction normal to the surface plane with a harmonic excitation. The solution is frequency dependent and is not suitable for transient analysis. A more adequate approach is presented in [25] where the damping force can be represented by a network of frequency independent spring-damper elements, which have the same transfer function of the initial solution. Replacing each of the spring-damper elements by its electrical equivalent, allows the modeling of the damping force for large displacements as a series of nonlinear inductances and resistances controlled by the displacement $x$. The values for the resistors and inductors [27] are

$$
\begin{aligned}
R_{m, n} & =(m n)^{2}\left(\frac{m^{2}}{w^{2}}+\frac{n^{2}}{l^{2}}\right) \frac{\pi^{6} d(x)^{3} Q_{\mathrm{pr}}\left(d(x), p_{a}\right)}{768 l w \eta} \\
L_{m, n} & =(m n)^{2} \frac{\pi^{4} d(x)}{64 l w p_{a}}
\end{aligned}
$$

where $m$ and $n$ are odd integers and $w$ and $l$ are the width and length of the surfaces (see Fig. 1), respectively.

As we are interested in understanding the behavior of the microstructure for displacements between the static pull-in limit and full gap, a large displacement model is needed. In order to model large displacements, the gap $d$ is made dependent of the displacement $\left(d(x)=d_{0}-x\right.$, with $d_{0}$ being the initial displacement). The Knudsen number (that models the rarefaction effects) is also included as displacement dependent parameter $\left(K_{n}=(\lambda) /(d(x))\right.$, where $\lambda$ is the gas mean free path) and accounted for in the model trough the relative flow rate coefficient

$$
Q_{\mathrm{pr}}=1+9.638\left(K_{n}\right)^{1.159}
$$

In surface-micromachined structures the flow passing the damper circumference has a significant effect on the damping coefficient due to the small thickness dimension, i.e., the length and width of the damper are comparable with the film thickness (gap size) [28], [29]. This is often referred to as the border effect and significantly changes the damping coefficient. For a surface width-to-gap size ratio as high as 20 , the damping force is still 35\% higher than predicted by (3), [28]. The border effects can be included in the analytically derived squeeze-film model using a modified surface length, $l_{0}=l+\Delta l$, and surface width, $w_{0}=w+w$. From [29], the effective elongation for a parallel-plate configuration with linear movement is given by

$$
\begin{gathered}
l_{0}=l+1.3 d \\
w_{0}=w+1.3 d
\end{gathered}
$$

resulting in a modified length and width that must be used in (3) to include the border effects in the damping model.

2) Large-Signal Model: A practical model should include system properties such as hysteresis of the pull-in [7] and the effect of stoppers. Therefore, the various physical parts of the system should be separately specified. As each of the RL sections presented before behaves like a first-order system with variable gain and time constant, the total damping force can be modeled as the sum of several damping forces of the type

$$
F_{m, n}=\frac{\dot{x}-\dot{F}_{m, n} L_{m, n}(x)}{R_{m, n}(x)} .
$$

Equation (1) can now be rewritten as

$$
F_{m}+F_{1,1}+F_{1,3}+F_{3,1}+\cdots+F_{m, n}+F_{k}=F_{\text {elec}} \cdot
$$

Adopting the notation $\dot{X}=f(X, V)$ yields for the overall nonlinear system the following equation of motion:

$$
\begin{aligned}
\dot{x} & =y \\
\dot{y} & =-\frac{1}{m}\left[\left(F_{1,1}+F_{3,1}+\cdots+F_{m, n}\right)+k x-\frac{1}{2} C(x) V^{2}\right] \\
\dot{F}_{1,1} & =\frac{y-F_{1,1} R_{1,1}(x)}{L_{1,1}(x)} \\
\dot{F}_{3,1} & =\frac{y-F_{3,1} R_{3,1}(x)}{L_{3,1}(x)} \\
\dot{F}_{m, n} & =\frac{y-F_{m, n} R_{m, n}(x)}{L_{m, n}(x)}
\end{aligned}
$$

where $x$ denotes the displacement, $y$ the velocity and $\dot{F}_{m, n}$ the time-derivative of the associated $F_{m, n}$ damping force. This approach clearly shows that the full dynamics of the system can be implemented in any simulation program using simple integrators and displacement controlled parameters. Moreover, it canbe simulated at the system level. A Simulink model is used to numerically solve the equation of motion [22].

\section{B. Phase Portrait}

A phase portrait is a plot of multiple trajectories in terms of state variables corresponding to different initial conditions in the same phase space [30]. It gives a qualitative view on the behavior of the system. For the microstructure the state variables are the displacement, $x$, and the velocity, $y$. The phase space is two-dimensional (2-D) with the displacement in the horizontal axis and the velocity in the vertical axis.

To design the phase portrait of the MEMS device, the nonlinear equation of motion is needed. The rather complex equation of motion in (9) can be simplified if low frequencies $(<100 \mathrm{kHz})$ are assumed. Considering the dimensions of the microstructure and the damping medium used, the cut-off frequency is in the order of the few megahertz [31], which makes this assumption valid. As a consequence, the spring component (the in-phase component of the squeeze-film solution due to compressibility) is neglected, and the equation of motion of the MEMS devices becomes

$$
\begin{aligned}
& \dot{x}=y \\
& \dot{y}=\frac{1}{m}\left(\frac{C_{0} d_{0} V^{2}}{2\left(d_{0}-x\right)^{2}}-k x-b(x) y\right)
\end{aligned}
$$

where $C_{0}$ is the initial zero-displacement capacitance. This device has the movable arm connected between two fixed electrodes and therefore, two gas films are present. Each film must be modeled separately since the gap distances are different $\left(d_{\text {left }}(x)=d_{0}-x\right.$ and $\left.d_{\text {right }}(x)=d_{0}+x\right)$, resulting in a nonlinear damping coefficient $b(x)$ given by

$$
b(x)=b_{\text {left }}(x)+b_{\text {right }}(x)
$$




\section{Legend}

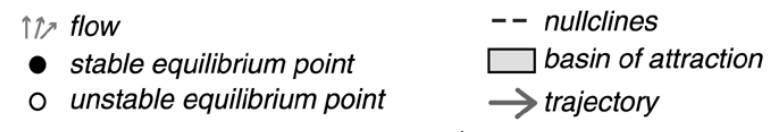

a)
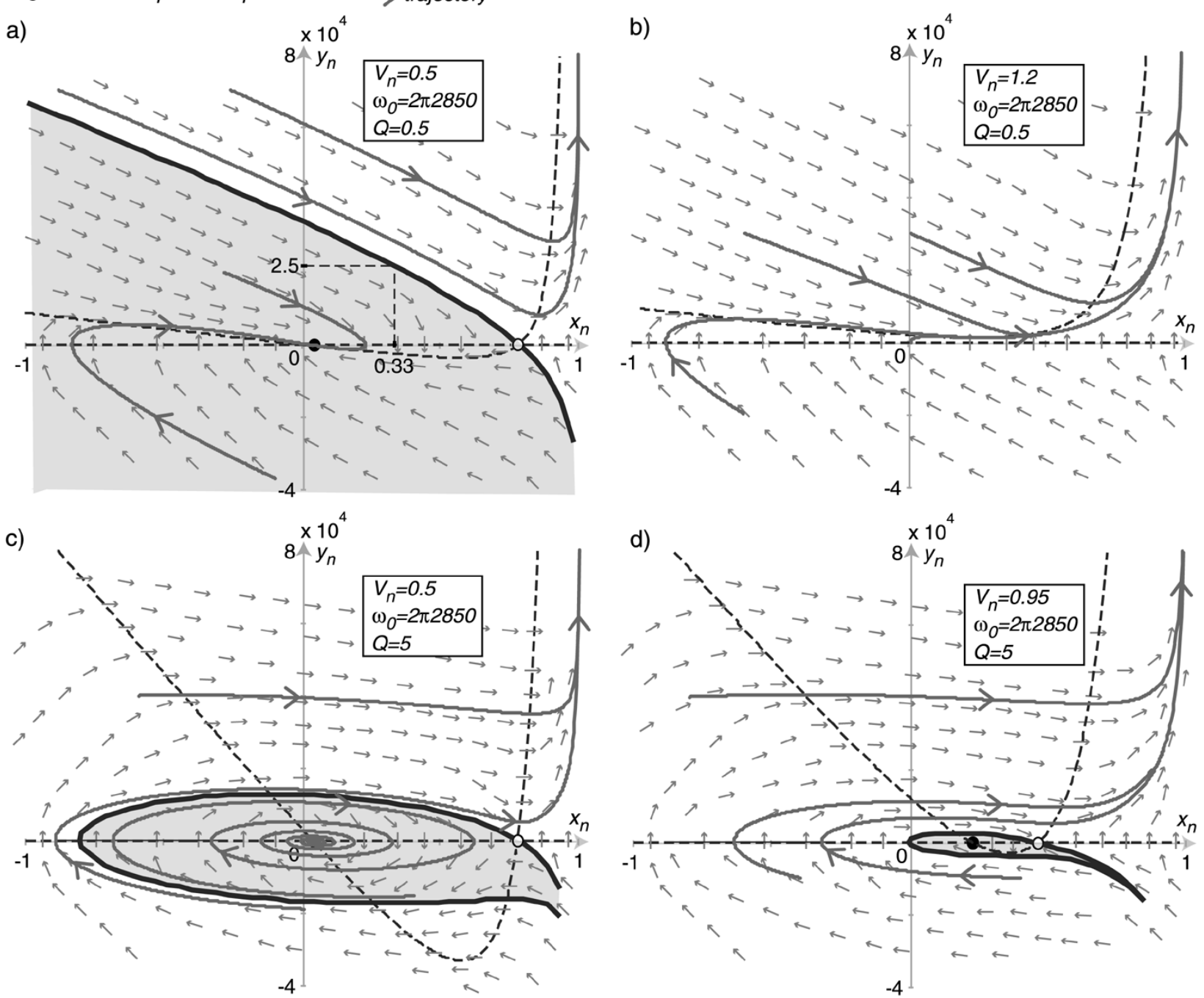

Fig. 2. Generic parallel-plate MEMS phase portraits. (a) $Q=0.5$ and $V_{n}=0.5$, (b) $Q=0.5$ and $V_{n}=1.2$, (c) $Q=5$ and $V_{n}=0.5$ and (d) $Q=5$ and $V_{n}=0.95$.

where $b_{\text {left }}(x)$ and $b_{\text {right }}(x)$ are determined by using (3)

$$
\begin{aligned}
& b_{\text {left }}(x) \\
& \quad=\sum_{m, n=\text { odd }} \frac{1}{R_{m n}} \\
& \quad=\sum_{m, n=\text { odd }} \frac{768 l_{0} w_{0} \eta}{\pi^{6} d_{\text {left }}^{3} Q_{\mathrm{pr}}\left(d_{\text {left }}, p_{a}\right)(m n)^{2}\left(\frac{m^{2}}{w_{0}^{2}}+\frac{n^{2}}{l_{0}^{2}}\right)} \\
& \quad b_{\text {right }}(x) \\
& \quad=\sum_{m, n=\text { odd }} \frac{1}{R_{m n}} \quad 768 l_{0} w_{0} \eta \\
& =\sum_{m, n=\text { odd }} \frac{\pi^{6} d_{\text {right }}^{3} Q_{\mathrm{pr}}\left(d_{\text {right }}, p_{a}\right)(m n)^{2}\left(\frac{m^{2}}{w_{0}^{2}}+\frac{n^{2}}{l_{0}^{2}}\right)}{}
\end{aligned}
$$

Equation (10) can be reformulated into a more convenient form, to emphasize the main parameters characterizing the dynamics of the system

$$
\begin{aligned}
& \dot{x}=y \\
& \dot{y}=\frac{1}{2} \frac{C_{0} d_{0}}{m\left(d_{0}-x\right)^{2}} V^{2}-\frac{\omega_{0}}{Q(x)} y-\omega_{0}^{2} x
\end{aligned}
$$

where $\omega_{0}=\sqrt{(k / m)}$ is the natural resonant frequency of the system and $Q(x)=\sqrt{(k m / b(x))}$ is the quality factor. A more generic representation of the parallel-plate case is achieved, irrespective of the numerical values of the coefficients involved, if a normalization of the state variables is performed. Both the displacement and velocity are normalized with respect to the initial gap spacing $d_{0}$

$$
x_{n} \equiv \frac{x}{d_{0}}[-], \quad y_{n} \equiv \frac{y}{d_{0}}\left[s^{-1}\right] .
$$




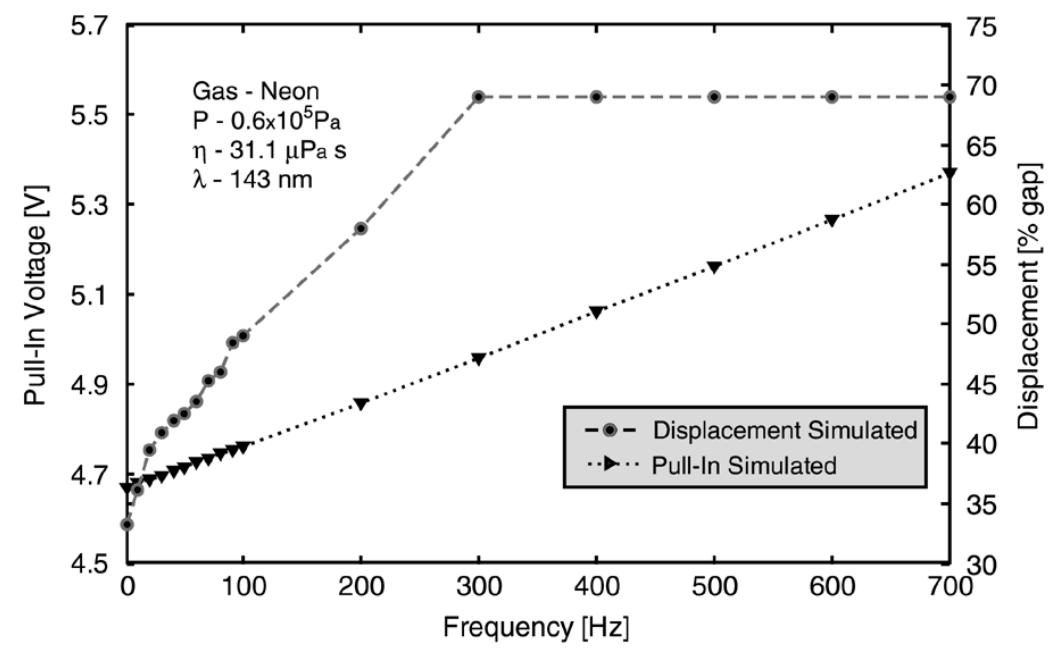

Fig. 3. Simulated dynamic pull-in voltage and dynamic pull-in displacement.

TABLE I

MAIN Microactuator PARAMETERS AND GAS PROPERTIES

\begin{tabular}{|l|c|l||c||}
\hline Device Parameters & Value & Gas Properties (Neon) & Value \\
\hline Mass $(m)$ & $2.3 \mu \mathrm{g}$ & Pressure & $0.6 \times 10^{5} \mathrm{~Pa}$ \\
Mechanical spring $(k)$ & $2.877 \mathrm{~N} / \mathrm{m}$ & Viscosity $(\eta)$ & $3.11 \times 10^{-5} \mathrm{Pa.s}$ \\
Resonance frequency $\left(f_{0}\right)$ & $5.7 \mathrm{kHz}$ & Mean free path $(\lambda)$ & $143 \mathrm{~nm}$ \\
Initial gap distance $\left(d_{0}\right)$ & $2 \mu \mathrm{m}$ & & \\
$C_{0}$ (initial zero-displacement & $157 \mathrm{fF}$ & \\
actuation capacitor) & $10.6 \mu \mathrm{m}$ & \\
Damper width $(w)$ & $280 \mu \mathrm{m}$ & \\
Damper length $(l)$ & $4.65 \mathrm{~V}$ & & \\
Predicted pull-in voltage & 40 & & \\
Total $n^{\circ}$ of arms & & \\
\hline
\end{tabular}

The voltage is normalized with respect to the pull-in voltage [7]

$$
V_{n}=\frac{V}{\sqrt{\frac{8}{27}} d_{0} \sqrt{\frac{k}{C_{0}}}} .
$$

Using the normalized variables and assuming a constant damping coefficient, the normalized equation of motion becomes

$$
\begin{aligned}
& \dot{x}_{n}=y_{n} \\
& \dot{y}_{n}=\frac{4}{27} \frac{\omega_{0}^{2}}{\left(1-x_{n}\right)^{2}} V_{n}^{2}-\frac{\omega_{0}}{Q} y_{n}-\omega_{0}^{2} x_{n}
\end{aligned}
$$

For the construction of the phase portrait the nullclines must be first determined. The nullclines correspond to the set of curves of the motion of the proof mass where the time derivative of one component of the state variables is zero. By making $\dot{x}_{n}=0$ and $\dot{y}_{n}=0$ in (16) the following nullclines result:

$$
\begin{aligned}
& y_{n}=0 \\
& y_{n}=\frac{Q}{\omega_{0}}\left(\frac{4}{27} \frac{\omega_{0}^{2}}{\left(1-x_{n}\right)^{2}} V_{n}^{2}-\omega_{0}^{2} x\right) .
\end{aligned}
$$

The displacement of the micromechanical device is physically constrained to the interval $-1 \leq x_{n} \leq 1$ due to the differential capacitor scheme. Since the important parameters are the quality factor, $Q$, and the applied voltage, $V_{n}$, phase portraits are constructed for different parameters settings in order to capture the nonlinear dynamic behavior of the generic micromechanical device. Fig. 2 shows the phase portraits.

Pull-in marks the boundary between a stable and an unstable operating mode. In the conventional static pull-in analysis, the parameters of interest are the voltage at which stability is lost and the maximum achievable displacement. In a dynamic pull-in analysis three issues are important: the motion described by the device, the time that it takes to hit the counter-electrode and the dynamic conditions that lead to loss of stability. An analysis of the phase portraits in Fig. 2 reveals that all the trajectories with initial values within the so-called basin of attraction tend to end at the equilibrium point, while all others collapse at the counter-electrode (dynamic pull-in situation).

The initial conditions of the system are thus a very important aspect of dynamic pull-in and dictate whether the system loses its stability as compared to the definition of static pull-in. The phase portraits show that dynamic pull-in occurs for all the state points outside the basin of attraction. This means that for voltages lower than $V_{\mathrm{pi}}$, the structure can still collapse. However, at the static pull-in displacement at $x_{n}=1 / 3$, the system would be within the basin of attraction for velocities $y_{n}<2500 \mathrm{~s}^{-1}$ in the case of a $Q=0.5$ and $V_{n}=0.5$ [see Fig. 2(a)]. The quality factor of the device is an important parameter in this case. For low-Q devices $(Q<0.5)$, and because there are no oscillations, the basin of attraction is larger than for the case of high-Q devices, where the oscillations strongly contribute to loss of stability. 


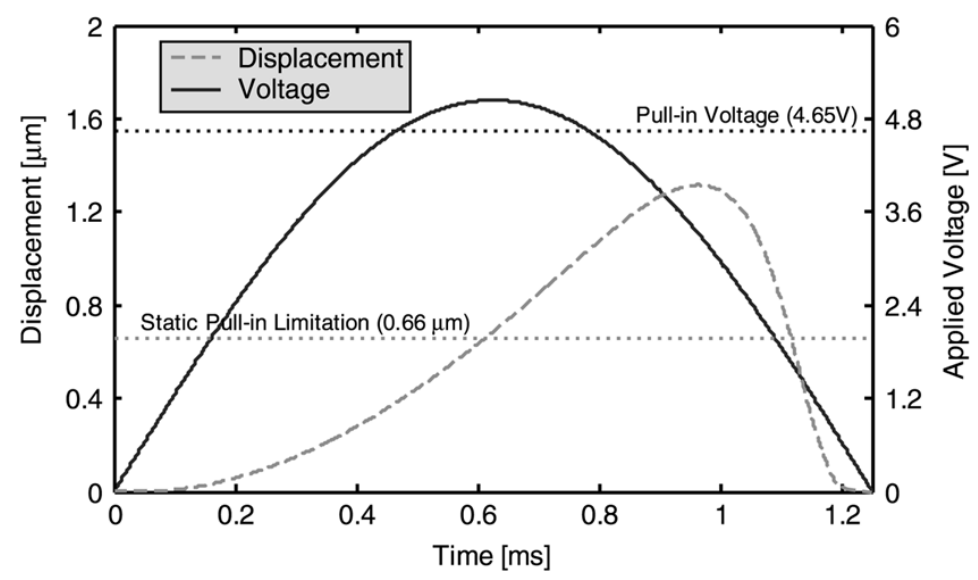

Fig. 4. Simulation results for a $400 \mathrm{~Hz}$ sine wave.

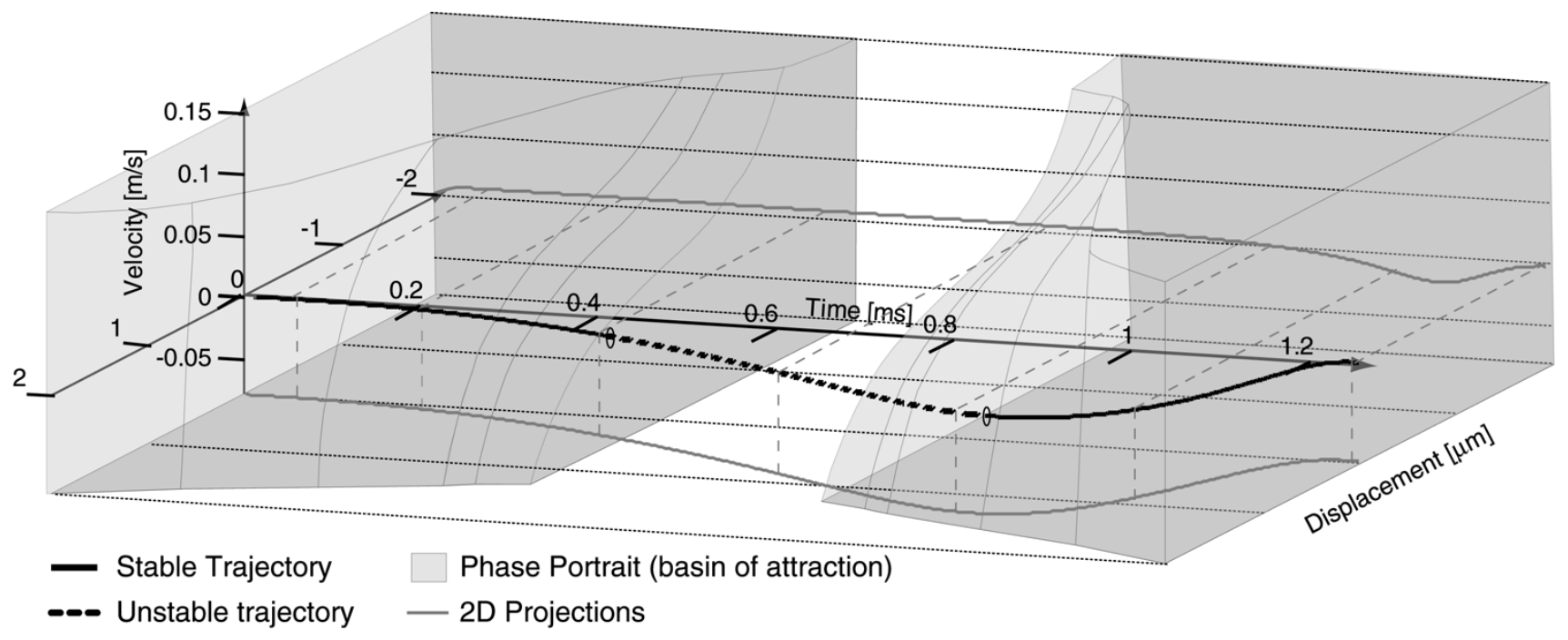

Fig. 5. Three-dimensional visualization of the phase portrait changes with voltage.

The important aspect are the changes in the phase portrait with voltage applied (both the nullclines and equilibrium points change with voltage). This indicates that the structure can dynamically go from a stable trajectory to an unstable one, or vice-versa, by a timely change in the voltage applied to slow-down the movement. Fig. 2(d) shows the phase portrait for a voltage $V_{n}=0.95$. When compared with Fig. 2(c) (same quality factor and $V_{n}=0.5$ ) it can be seen that the basin of attraction was strongly reduced and almost all state points lead to dynamic pull-in. This leads to the conclusion that a properly designed time-dependent voltage should be able to prevent the microstructure from losing stability and, consequently, displacements beyond static pull-in limitation can be achieved by dynamically applying a sufficiently high voltage level. Moreover, a sufficient damping force must be available to yield a practical window in time to change the voltage.

\section{Dynamic Displacement}

In the previous section it was concluded that, although the phase portrait of a MEMS changes with voltage applied with no stable point outside the basin of attraction, a point in the unstable region can nevertheless be reached without causing instability when using dynamic drive with an ac voltage of suffi- ciently high peak amplitude to escape the base of attraction and a rate of change higher than the change in motion of the structure to oppose and subsequently reverse motion after passage of that point and to reenter the base of attraction. For this purpose the voltage amplitude should be high enough to bring the structure out of the basin of attraction and of sufficient high frequency to compensate for the intrinsic dynamics of the MEMS. Using a large-signal model with the main micromechanical device parameters presented in Table I, simulations were performed to prove this concept. Sine waves of different voltage amplitudes and frequencies were applied to the device and the maximum achievable displacement and the voltage for which the structure loses stability (dynamic pull-in) were recorded. The results are shown in Fig. 3.

The simulation results confirm that the MEMS can be dynamically operated beyond the static pull-in displacement, providedthat the appropriate ac voltage is applied. To have a better insight on what is happening during a half period of a sine, simulations are carried out for the case of applying a $400 \mathrm{~Hz}$ sine wave voltage with an amplitude of $5.04 \mathrm{~V}$. The results are shown in Fig. 4 and indicate that, although the static pull-in voltage and the static pull-in displacement are exceeded, the structure does not go into pull-in. 


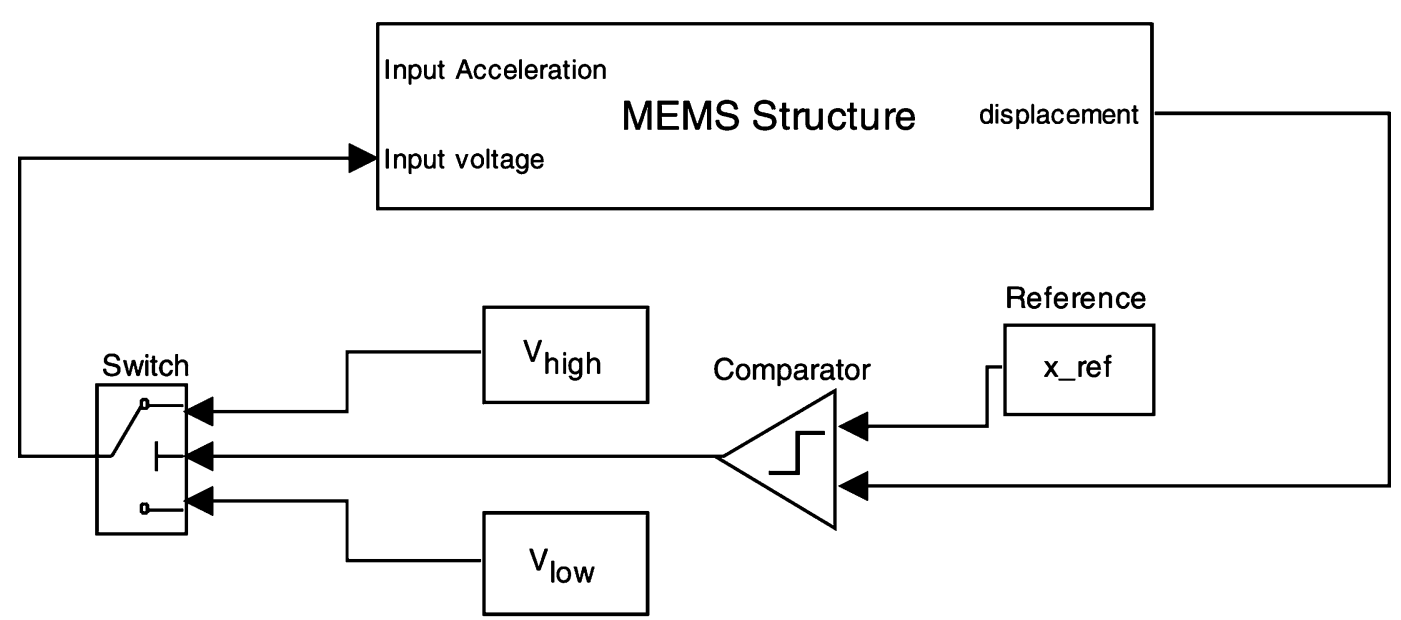

Fig. 6. Block diagram of the on-off approach.

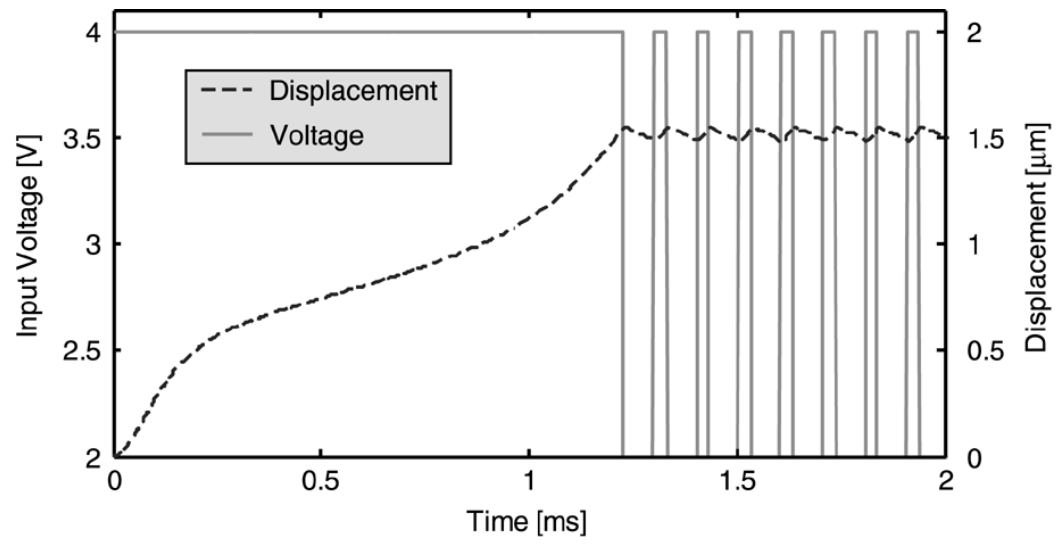

Fig. 7. Simulink simulations of the on-off control scheme.

An even more revealing exercise is the computation of the phase portrait along a time axis for the voltage changes. Keeping with the same example ( $400 \mathrm{~Hz}$ sine wave), the phase portrait of the MEMS device is computed for several points (different voltages). Combining the phase portraits yields a 3-D image of the basin of attraction. If the trajectory of the device is included (for an applied half-sine at $400 \mathrm{~Hz}$ ), the 3-D graph of Fig. 5 results. This 3-D image shows what has been previously stated. Initially the device trajectory is inside of the basin of attraction but, with the increasing of the voltage, the structure leaves the basin of attraction (unstable trajectory). A movement toward the counter-electrodes can be observed after leaving the basin of attraction. However, since the rate of decrease in voltage is higher than the displacement changes, the unstable trajectory reenters the basin of attraction and returns to a stable trajectory. This can be repeated using a periodic voltage and demonstrates the possibility of trajectories beyond the static pull-in limitation without going to pull-in, thus maintaining a dynamic trajectoryoutside the static pull-in stability boundary without collapsing on the counter-electrode. These experiments demonstrate the ability to generate an actuator motion that includes the part of the gap that used to be off-limits, however, does not enable stable positioning within that part of the gap. The technique is open-loop (i.e., no position information is used in a feedback configuration). This dynamic drive approach can be extended to a tech-
TABLE II

MiCROACTUATOR 2 PARAMETERS

\begin{tabular}{|l|c|}
\hline Device Parameters & Value \\
\hline Mass $(m)$ & $4.27 \mu \mathrm{g}$ \\
Mechanical spring $(k)$ & $1.29 \mathrm{~N} / \mathrm{m}$ \\
Resonance frequency $\left(f_{0}\right)$ & $2.77 \mathrm{kHz}$ \\
Initial gap distance $\left(d_{0}\right)$ & $2.25 \mu \mathrm{m}$ \\
$C_{0} \quad$ (initial zero-displacement & $141 \mathrm{fF}$ \\
actuation capacitor) & $10.6 \mu \mathrm{m}$ \\
Damper width $(w)$ & $282 \mu \mathrm{m}$ \\
Damper length $(l)$ & 72 \\
Total $n^{\circ}$ of arms & \\
\hline
\end{tabular}

nique for stable positioning of the microstructure to any position within the gap when using position information, which is demonstrated in the next section.

\section{Dynamic Voltage Drive: ON-OFf Closed-LooP}

The analysis of the nonlinear dynamics performed leads to a method for achieving stable displacements beyond the pull-in limitation, which is simpler and more flexible compared to feedback linearization and charge control (current drive) techniques. The basic idea is the comparison of the momentary actuator displacement being measured with a fixed desired displacement. Unlike feedback linearization, the applied voltage on the actu- 


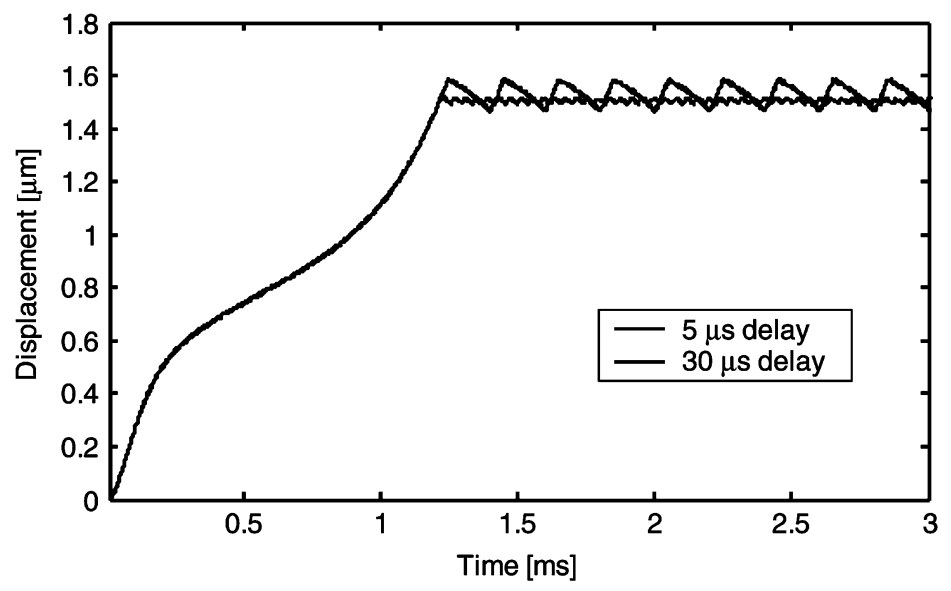

Fig. 8. Simulations of the on-off control scheme with different delay times.

ator is changed between two values according to the comparison result. A high level (in excess of the static pull-in voltage $V_{\mathrm{pi}}$ ), if the momentary actuator displacement is lower than the position setting, and a lower level (smaller than $V_{\mathrm{pi}}$ ), if the actuator displacement exceeds the set value. The only requirement for implementation is the sufficient fast measurement of the displacement of the microstructure. The surface micromachined devices used in this work are equipped with sets of differential sensing capacitors that can be used to measure the device displacement.

The concept is simple and similar to the on-off method used in linear control theory [32]. A block diagram of the proposed method is shown in Fig. 6. The method relies on the dynamics of the MEMS devices, namely the shift between stable and unstable trajectories (shift on the basin of attraction) by changing the applied voltage. This implies that the device must be overdamped or critically damped. In underdamped devices the inertial forces are not significantly counteracted by damping forces and the oscillatory regime typical of these devices compromises the method.

The voltage levels are not critical for proper operation. The high voltage level has to be initially higher than the pull-in voltage, but can be dynamically reduced to improve performance. The low voltage level must guarantee that before theswitching, the structure position is within the low-level voltage range of attraction. However, the difference between high and low level is affecting the device response and can be dynamically reduced to improve the performance.

These two aspects are essential for successful implementation of the method: switching and delay times in the control loop.

1) Time Delays in the Feedback Loop: During switching the structure continues the movement, and consequently an extra displacement results, which leads to a small ripple around the desired displacement. This effect is aggravated by any time delay introduced by the circuits.

Using a large-signal model for a 1-DOF actuator with the characteristics shown in Table II, a Simulink model was implemented to verify the proposed control method. The equivalent mechanical and estimated electrical noise sources are included in the model, and a time delay is introduced in the feedback loop. The introduced delay includes both the switching times and the circuit delays. Fig. 7 shows the simulated performance of the control loop for a delay of $15 \mu \mathrm{s}$. The reference displacement is set at $1.5 \mu \mathrm{m}$ and the voltage levels are defined as: $V_{\text {high }}=4 \mathrm{~V}$ and $V_{\text {low }}=2 \mathrm{~V}$. The simulated MEMS actuator has a theoretical static pull-in voltage at $V_{\mathrm{pi}}=3.820 \mathrm{~V}$ and the stoppers are placed at $2 \mu \mathrm{m}$ in a $2.25 \mu \mathrm{m}$ gap.

Additional simulations were performed using different time delays with the results shown in Fig. 8. As predicted, the ripple depends on the time delay and the displacement beyond the reference displacement value increases with delay time. Despite its simplicity, these results predict the feasibility of the on-off control method. The main constrains are the ripple in the microstructure position around the set value due to time delays in the readout circuits. According to simulations, the control remains effective for delays as long as $30 \mu$ s. Since switching time in a comparator is lower than $1 \mu \mathrm{s}$, the bottleneck for actual implementation is the phase characteristics of the readout circuit.

\section{MicrostruCture FABRICATION}

The Bosch epi-poly process was used for the fabrication of the actuators [33], [34] used in this work. This process is very suitable for the fabrication of relatively thick and high aspect ratio free-standing beams on top of a silicon wafer. Epitaxial growth at about $700 \mathrm{~nm} / \mathrm{min}$. is used to yield a 10.6- $\mu \mathrm{m}$-thick polysilicon layer on top of a dielectric oxide. After deposition the polysilicon layer is patterned using deep-reactive ion etching (DRIE). Microstructures are subsequently released by selectively etching the underlying dielectric sacrificial layer using the DRIE holes as access channel.

\section{A. Actuator 1}

The structure used for the verification of the nonlinear dynamic analysis is the 1-DOF structure shown in Fig. 9 (the main device parameters are presented in Table I). It consists of four folded beams, $375 \mu \mathrm{m}$ long and $2.8 \mu \mathrm{m}$ wide, connected to a rigid central bar of $1000 \mu \mathrm{m}$ length. A set of interdigitated parallel-plate electrodes with an initial gap of $2 \mu \mathrm{m}$ between fixed and movable structures are used for actuation. The measurement of the displacement is implemented by capacitive sensing of the distance in two other sets of electrodes. Stoppers located on either side of the rigid bar limit the movement after pull-in is reached to avoid an electric short-circuit. 

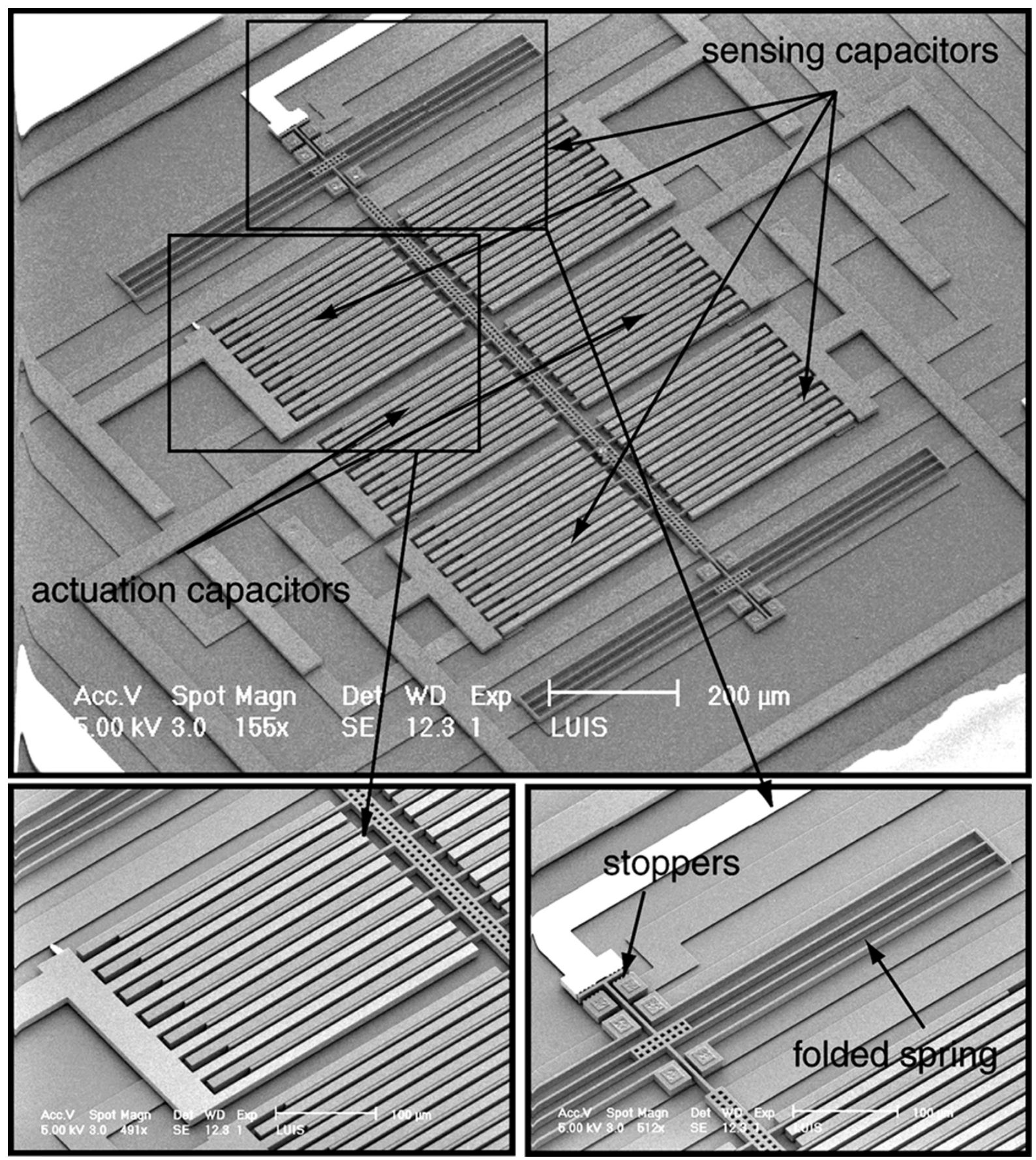

Fig. 9. Actuator 1 microphotograph.

\section{B. Actuator 2}

The second actuator fabricated is a remodeled version of the first device. It is a laterally movable structure (with two central bars) with folded beam suspension at both ends and electrodes extending perpendicular to the axial direction. Several sets of electrodes in the same plane are used. Two are for electrostatic actuation in the direction normal to the electrode area, and the rest are used for capacitive displacement measurement. A SEM photograph of the structure is shown in Fig. 10 (the main device parameters are presented in Table II).

The movable arms of this device are double-sided clamped, which reduces the arms bending after reaching pull-in as com- pared to actuator 1 (single-sided arms). There are also more arms available for capacitance readout, which increases the displacement-to-capacitance sensitivity and therefore improves the detection limit of the capacitance measurement. Comparatively, the second actuator has a bigger mass, a smaller equivalent mechanical spring (actuator 1 has eight pairs of folded beams, while actuator 2 only has four pairs) and a higher capacitance change due to displacement, resulting in a device with a lower resonant frequency and a lower $\mathrm{Q}$ value at ambient pressure (due to the high number of arms that also contribute to the increase of the damping coefficient). This actuator is used in the implementation of the on-off closed-loop method. 


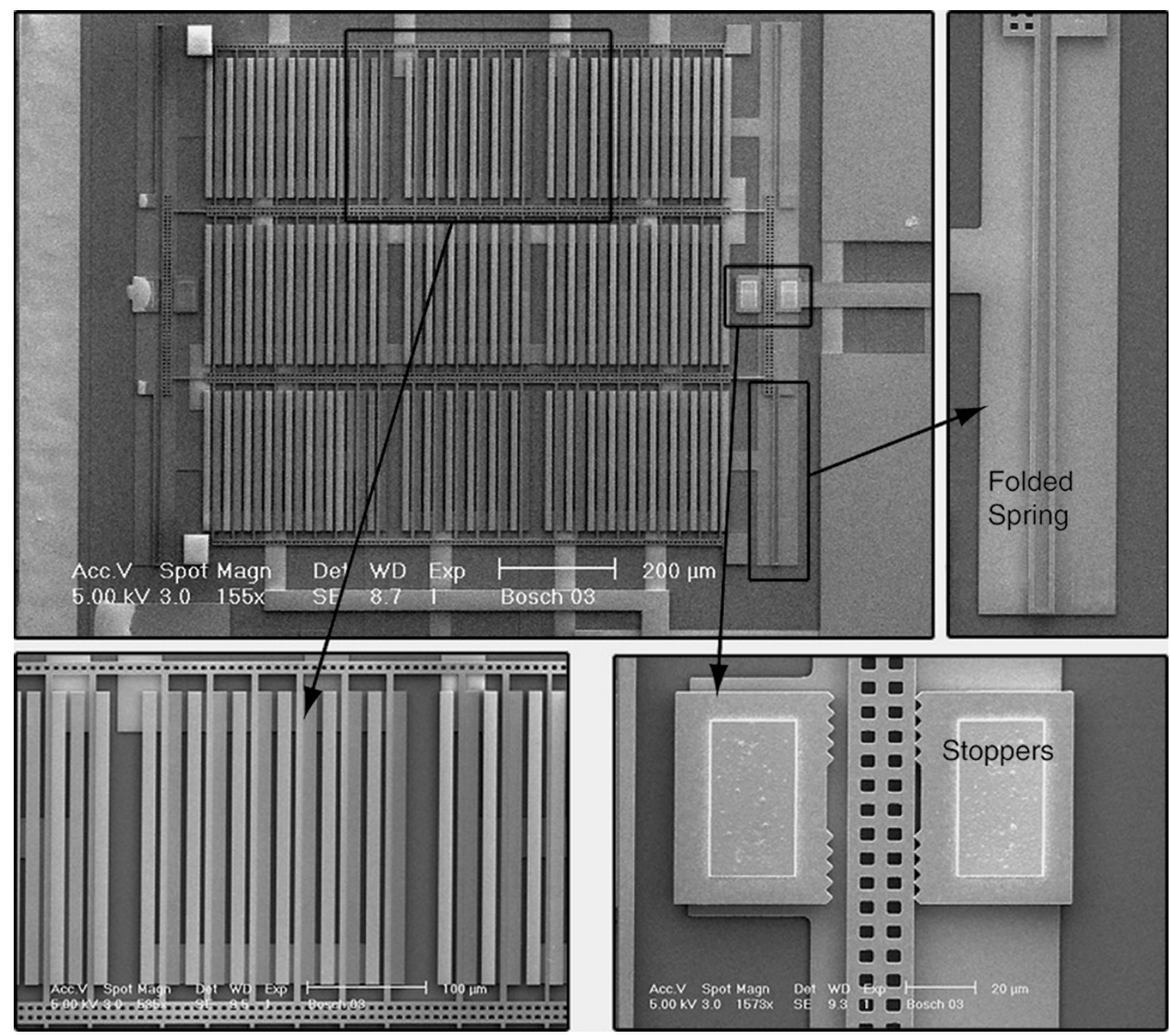

Fig. 10. SEM photograph of actuator 2 .

\section{EXPERIMENTAL RESULTS}

Since the readout electronic circuit is a critical component for the implementation of the closed-loop on-off control, the differential capacitance readout circuit is presented here. A differential output circuit [35], [36] has been used to measure the displacement on the fabricated MEMS actuators. The fabricated MEMS devices have differential sensing capacitors and allow for capacitive displacement detection.

A differential sense interface is used with the sensing stationary electrodes connected to the differential input of the charge amplifier and the drive signal applied to the common movable central point. A schematic of the differential output circuit is shown in Fig. 11. The circuit is composed of three main blocks: a buffer amplifier, two charge amplifiers and an amplitude demodulator. The buffer amplifier sets the gain for the carrier input voltage, the charge amplifier modulates the amplitude of the carrier signal proportional to the capacitance changes and the demodulator shifts the modulated carrier signal to the (mechanical) baseband.

The differential sensing circuit depicted in Fig. 11 was implemented at the PCB level. Commercially available transconduc- tance amplifiers [37] were used in the high-frequency path. The amplitude demodulator stage was implemented using a 1496 Motorola demodulator. The bandwidth of the low-pass filter was set at $200 \mathrm{kHz}$, resulting in total estimated circuit delay at $5 \mu \mathrm{s}$.

\section{A. Dynamic Pull-In Measurements}

Actuator 1 was used to experimentally validate the nonlinear dynamic analysis, especially the open-loop dynamic displacement. Similarly to the simulations performed previously, sine waves of different voltage amplitudes and frequencies were applied to the device. A comparison between measured and simulated values is presented in Fig. 12.

Displacements up to $70 \%$ of the gap were measured using ac operation in the $300-700 \mathrm{~Hz}$ range. These experimental results prove some of the dynamic pull-in ideas previously introduced in this paper. Although no position control over the applied voltage is performed in these experiments, displacements beyond the static pull-in limitation were achieved using peak voltage levels higher than the static pull-in voltage, $V_{\mathrm{pi}}$. These results have an immediate repercussion on the commonly used 


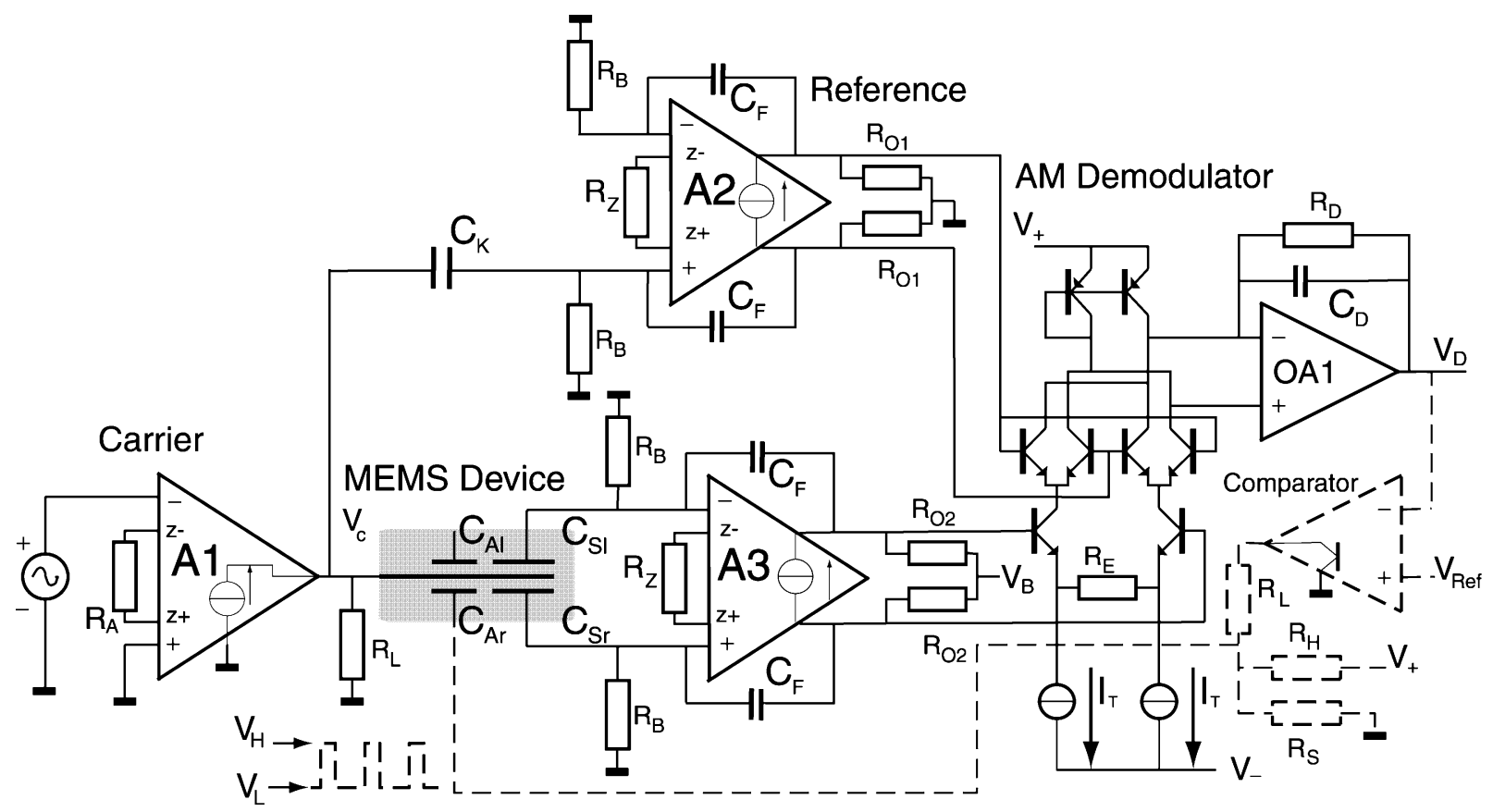

Fig. 11. Schematic of the differential sense interface.

a)

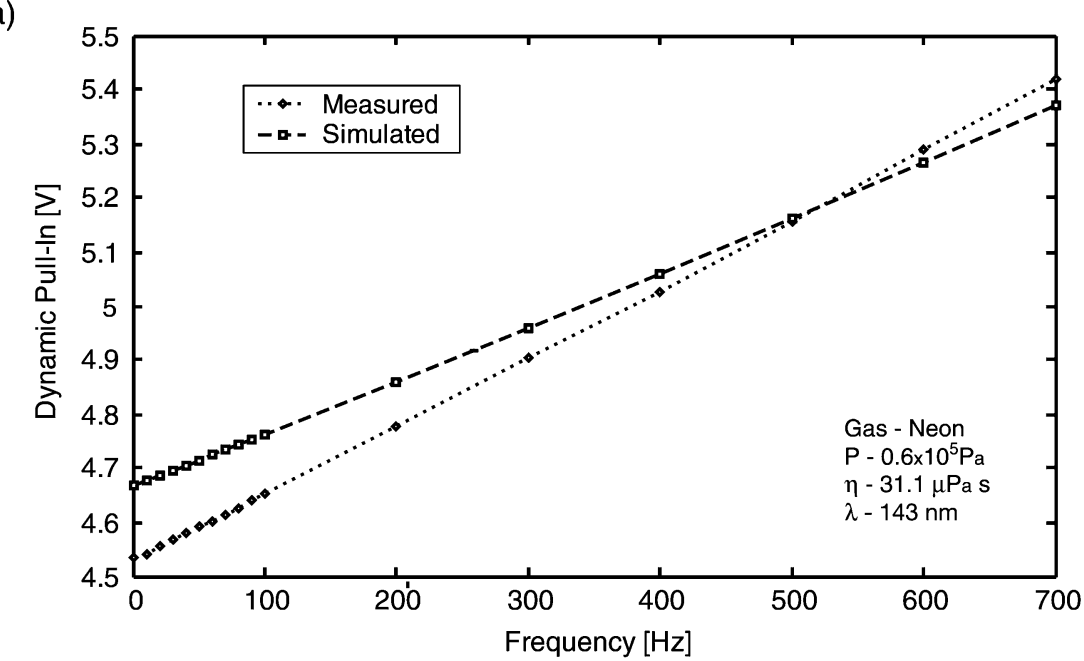

b)

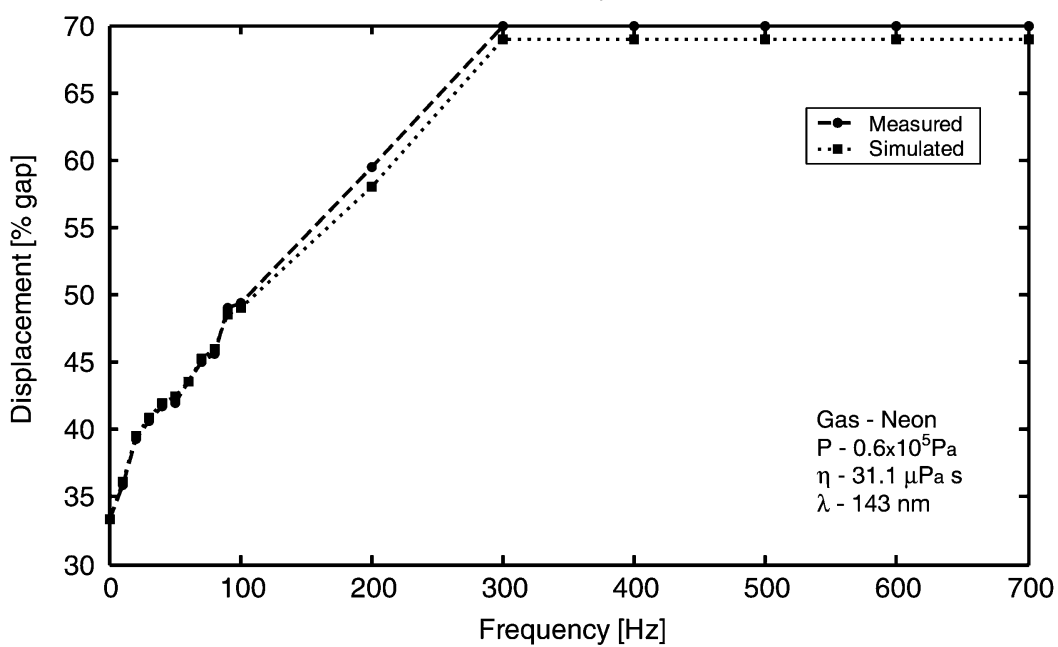

Fig. 12. Measured and simulated (a) dynamic pull-in voltage and (b) dynamic pull-in displacement. 


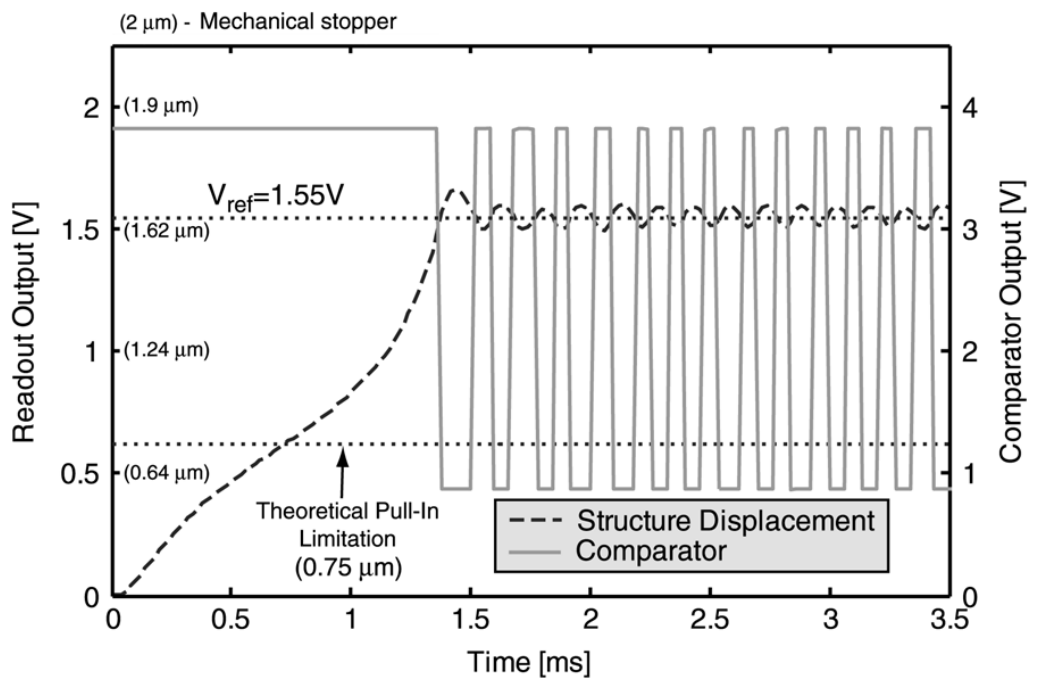

Fig. 13. Measured operational details of the on-off method.

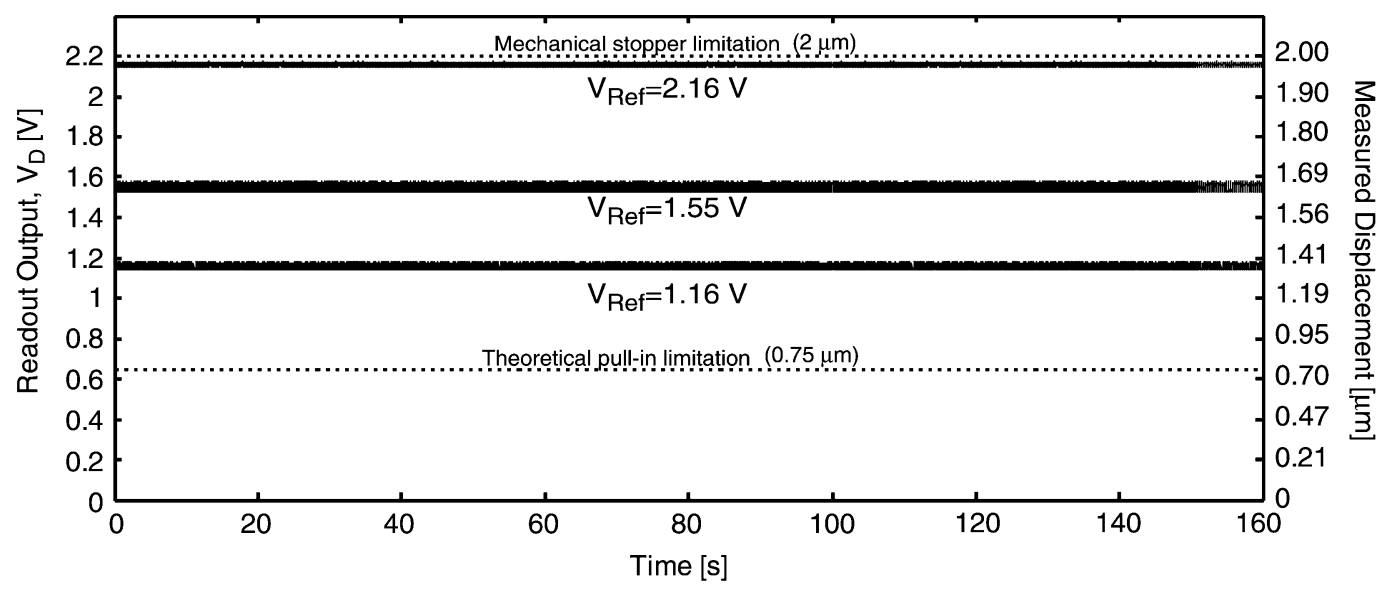

Fig. 14. Measured voltage-drive stable operation beyond the theoretical pull-in limitation.

sentence about parallel-plate electrostatic actuated MEMS devices [14]: "The pull-in instability limits the travel distance of elastically suspended parallel-plate electrostatic microactuators to about $1 / 3$ of the undeflected gap distance". This sentence should be reformulated to: "Static pull-in limits the stable displacement range of a $d c$-voltage-operated parallel-plate-actuated microstructure to a maximum of $1 / 3$ of the full gap."

\section{B. Stable Extended Travel Range}

Operation of the on-off method has been experimentally verified using actuator 2 structures (hermetically sealed and filled with neon at $0.6 \times 10^{5} \mathrm{~Pa}$ by manufacturer). A comparator was introduced in the readout circuit to close the feedback loop (see dashed components in Fig. 10). The voltage $V_{\mathrm{D}}$, which is proportional to the actuator displacement, is compared with the reference value, $V_{\text {Ref }}$, and the drive feedback signal varies between $V_{\mathrm{H}}$ and $V_{\mathrm{L}}$ according to the comparator output. $V_{\mathrm{H}}$ and $V_{\mathrm{L}}$ are set by

$$
V_{H}=\frac{R_{S}}{R_{S}+R_{H}} V_{+} \quad \text { and } \quad V_{L}=\frac{R_{S} / / R_{L}}{\left(R_{S} / / R_{L}\right)+R_{H}} V_{+} \cdot
$$

The mechanical stoppers limit the actuator displacements to $2 \mu \mathrm{m}$ in a $2.25-\mu \mathrm{m}$ gap. The readout output voltage, $V_{\mathrm{D}}$, ranges from 0 (zero displacement) until $2.2 \mathrm{~V}(2 \mu \mathrm{m}$ displacement $)$ and at $1 / 3$ of the gap $(0.75 \mu \mathrm{m})$ the output readout voltage is 0.62 V. First, the operational details of the method were verified by measuring the comparator output and actuator displacement at the time the feedback loop is closed. Measured results are shown in Fig. 13.

Stable positioning at a voltage selected operated point beyond the static pull-in limitation is achieved in agreement with simulations. Additional measurements were performed to check the stability of the method over time and at different reference positions. Stable operation over the full available gap $(2 \mu \mathrm{m})$ is observed. The results for three different points are shown in Fig. 14.

Although the stoppers limit operation up to $89 \%$ of gap, full range operation can be achieved with this approach on devices without mechanical limitations. At very small gaps, the damping force is huge due to the very narrow channels available for gas flow. This damping force slows even further the structure motion, thus improving the dynamic device response when operated with the on-off method. The high damping coefficient at very small gaps is in fact expected to enhance operation. 


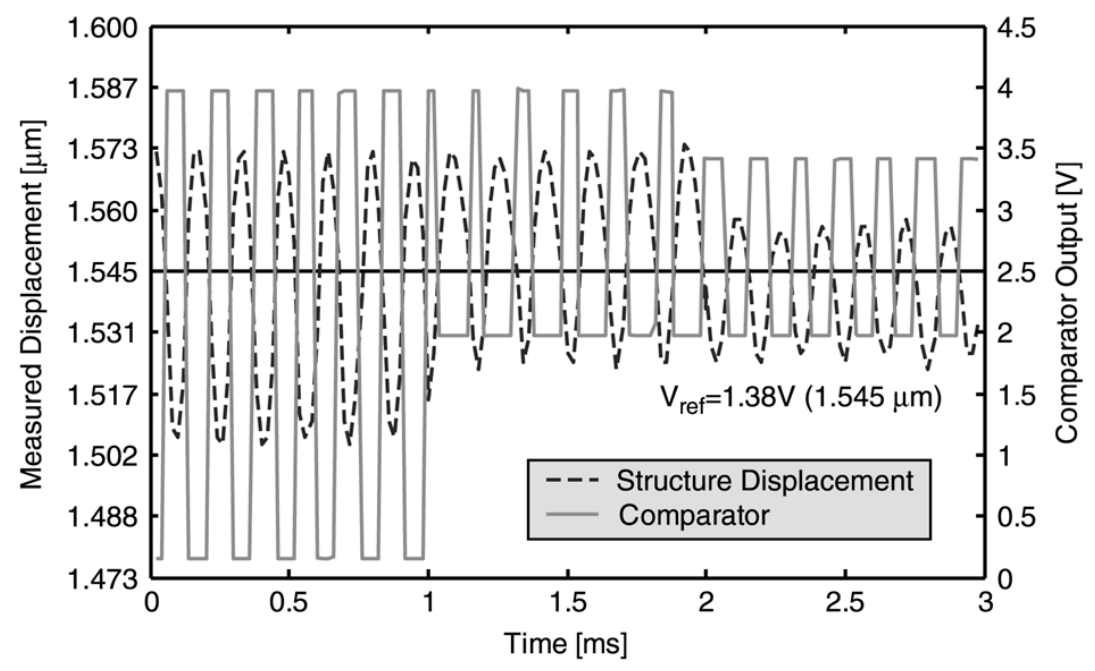

Fig. 15. Measured changes in ripple amplitude with different voltage levels.

TABLE III

COMPARison Between Reported Methods for Extending the RANGe of Parallel-Plate Electrostatic Actuators

\begin{tabular}{|c|c|c|c|c|}
\hline \multicolumn{2}{|c|}{ Method } & $\begin{array}{c}\text { Maximum } \\
\text { displacement }\end{array}$ & Advantages & Disadvantages \\
\hline \multicolumn{2}{|c|}{ Geometry leverage [14] } & $\cong 90 \%$ & $\begin{array}{l}\text { - Simple } \\
\text { - Does not require a sensing } \\
\text { mechanism }\end{array}$ & $\begin{array}{l}\text { - Increased actuation voltage } \\
\text { - Increased device area }\end{array}$ \\
\hline \multicolumn{2}{|c|}{ Series capacitance $[16]$} & $\cong 65 \%$ & $\begin{array}{l}\text { - Simple } \\
\text { - Does not require a sensing } \\
\text { mechanism }\end{array}$ & $\begin{array}{l}\text { - Reduce travel due to parasitic } \\
\text { capacitances and residual charge } \\
\text { - Increased actuation voltage }\end{array}$ \\
\hline \multirow{2}{*}{ Current drive } & [19] & $\cong 65 \%$ & \multirow{2}{*}{$\begin{array}{l}\text { - No increase in device area } \\
\text { - Does not require a sensing } \\
\text { mechanism }\end{array}$} & \multirow{2}{*}{$\begin{array}{l}\text { - Charge pull-in due to parasitic } \\
\text { capacitances and tip-in instability } \\
\text { - On-board current sources and } \\
\text { charge control circuitry } \\
\text { - Increased actuation voltage }\end{array}$} \\
\hline & {$[20]$} & $83 \%$ & & \\
\hline \multicolumn{2}{|c|}{ Feedback Linearization [23] } & $74 \%$ & $\begin{array}{l}\text { - Does not require increased } \\
\text { actuation voltage }\end{array}$ & $\begin{array}{l}\text { - Complex } \\
\text { - Difficult implementation } \\
\text { - Sensing mechanism needed } \\
\end{array}$ \\
\hline \multicolumn{2}{|l|}{ This paper } & $89 \%$ & $\begin{array}{l}\text { - Simple } \\
\text { - Flexible } \\
\text { - } 100 \% \text { travel range if used } \\
\text { without stoppers } \\
\end{array}$ & $\begin{array}{l}\text { - Ripple (due to delay) } \\
\text { - Sensing mechanism needed }\end{array}$ \\
\hline
\end{tabular}

Finally, the voltage levels were dynamically adjusted to check its influence on the ripple. Results for the case of a reference voltage, $V_{\text {Ref }}=1.38 \mathrm{~V}(\cong 1.54 \mu \mathrm{m})$ are shown in Fig. 15. The figure clearly shows that the voltage levels are not critical for device operation, but adaptation during positioning does reduce the ripple (= positionluncertainty) of the on-off control. The best results obtained so far using the most suitable voltage level combination available yield a ripple of about $30 \mathrm{mV}(\cong 30 \mathrm{~nm})$. The main source of ripple is the time delay introduced by the readout. Actuator 2 has a mechanical-thermal noise (theoretical) of $\cong 56 \mathrm{pm}$, while the predicted readout noise referred to the input is about $\cong 180 \mathrm{pm}$. For ideal time delay the stability of the on-off approach would be limited by noise, and for Actuator 2 the stability would be $\cong 230 \mathrm{pm}$, which is equivalent to the ripple caused by a delay time of about $50 \mathrm{~ns}$. Therefore, only for a delay time around $50 \mathrm{~ns}$, the stability of the position is set by both the mechanical-thermal noise and the readout noise.

\section{CONCLUSION}

In this paper, the nonlinear dynamics of MEMS devices have been analyzed with special emphasis on the dynamics of pull-in. The motion of a microstructure actuated by a voltage is described by a set of nonlinear differential equations. When the dynamic behavior is considered, the loss of stability is more difficult to predict, as compared to a quasistatic analysis. Pull-in can happen for voltages lower than the static pull-in value, depending on the initial state variable values and external applied forces. This is of extreme importance for applications requiring a stable behavior. There are no analytical tools to predict these values, but by numerically solving the differential equation of motion the boundaries of stability can be found. The analysis of the nonlinear differential equation shows that the stability and trajectory described depend on the voltage applied. With a proper ac voltage, 1) sufficiently high-peak amplitude to reach 
the point and 2) sufficiently high frequency to overcompensate the rate of change of the microstructure motion, any point in the phase space can be encompassed (i.e., periodically reached).

The insight gained from this nonlinear analysis has led to a new technique for extending the positioning range of parallel-plate electrostatic actuators. Full-gap positioning is demonstrated using on-off control with capacitive position. The simplicity and flexibility of this approach highly facilitates implementation in parallel-plate based electrostatic actuator systems. Table III gives a comparative overview of this method and those previously reported in literature.

The voltage drive relies on the device dynamics and the dependence of the positioning ripple on the time delay introduced by the readout. Limitations are the need for displacement sensing and the fact that it can be used in low-Q devices only. In the present system, ripples as small as $30 \mathrm{~nm}$ over the full gap available have been achieved. Further studies are needed in order to improve the performance of the on-off method.

\section{ACKNOWLEDGMENT}

The authors acknowledge G. de Graaf and L. Mol for assistance in readout circuit design.

\section{REFERENCES}

[1] S. F. Bart, T. A. Lober, R. T. Howe, J. H. Lang, and M. F. Schlecht, "Design considerations for micromachined electric actuator," Sens. Actuators A, Phys., vol. A 14, pp. 269-292, 1988.

[2] T. Ono, D. Y. Sim, and M. Esashi, "Micro discharge and electric breakdown in a micro gap," J. Micromech. Microeng., vol. 10, pp. 445-451, 2000.

[3] E. Cretu, M. Bartek, and R. F. Wolffenbuttel, "Spectral analysis through electro-mechanical coupling," Sens. Actuators A, Phys., vol. A85, no. $1-3$, pp. 23-32, 2000.

[4] M. Wautelet, "Scaling laws in the macro-, micro-, and nanoworlds," Eur. J. Phys., vol. 22, pp. 601-611, 2001.

[5] H. A. C. Tilmans and R. Legtenberg, "Electrostatically driven vacuumencapsulated polysilicon resonators, Part 2, Theory and performance," Sens. Actuators A, Phys., vol. A 45, pp. 67-84, 1994.

[6] H. C. Nathanson, W. E. Newell, R. A. Wickstrom, and J. R. Davis, "The resonant gate transistor," IEEE Trans. Electron Devices, vol. 14, pp. 117-133, 1967.

[7] L. A. Rocha, E. Cretu, and R. F. Wolffenbuttel, "Analysis and analytical modeling of static pull-in with application to mems-based voltage reference and process monitoring," J. Microelectromech. Syst., vol. 13, pp. 342-354, 2004.

[8] S. T. Cho, K. Najafi, and K. D. Wise, "Internal stress compensation and scaling in ultrasensitive silicon pressure sensors," IEEE Trans. Electron Devices, vol. 39, pp. 836-842, 1992.

[9] P. M. Osterberg and S. D. Senturia, "M-Test: A test chip for MEMS material property measurement using electrostatically actuated test structures," J. Microelectromech. Syst., vol. 6, pp. 107-118, 1997.

[10] M. H. H. Nijhuis, T. G. H. Basten, Y. H. Wijnant, H. Tijdeman, and H. A. C. Tilmans, "Transient nonlinear response of "pull-in MEMS devices' including squeeze film effects," in Proc. Eurosensors XIII, 1999, pp. 729-732.

[11] L. M. Castañer and S. D. Senturia, "Speed-energy optimization of electrostatic actuators based on pull-in," J. Microelectromech. Syst., vol. 8, pp. 290-298, 1999

[12] S. Krylov and R. Maimon, "Pull-in dynamics of an elastic beam actuated by continuously distributed electrostatic force," Trans. Amer. Soc. Mechan. Eng., vol. 126, pp. 332-342, 2004.

[13] A. C. J. Luo and F.-Y. Wang, "Nonlinear dynamics of a micro-electromechanical system with time-varying capacitors," J. Vibr. Acoust., vol. 126, pp. 77-83, 2004.

[14] E. S. Hung and S. D. Senturia, "Extending the travel range of analog-tuned electrostatic actuators," J. Microelectromech. Syst., vol. 8, pp. 497-505, 1999.
[15] J. I. Seeger and S. B. Crary, "Stabilization of electrostatic actuated mechanical devices," in Proc. Tranducers'97, Chicago, IL, USA, Jun. 16-19, 1997, pp. 1133-1136.

[16] E. K. Chan and R. W. Dutton, "Electrostatic micromechanical actuator with extended range of travel," J. Microelectromech. Syst., vol. 9, pp. $321-328,2000$

[17] J. I. Seeger and S. B. Crary, "Dynamics and control of parallel-plate actuators beyond the electrostatic instability," in Proc. Tranducers'99, Sendai, Japan, Jun. 7-10, 1999, pp. 474-477.

[18] L. Castañer, J. Pons, R. Nadal-Guardia, and A. Rodríguez, "Analysis of the extended operation range of electrostatic actuators by current-pulse drive," Sens. Actuators A, Phys., vol. A 90, pp. 181-190, 2001.

[19] R. Nadal-Guardia, A. Dehé, R. Aigner, and L. M. Castañer, "Current drive methods to extend the range of travel of electrostatic microactuators beyond the voltage pull-in point," J. Microelectromech. Syst., vol. 11 , pp. 255-263, 2002.

[20] J. I. Seeger and B. E. Boser, "Charge control of parallel-plate, electrostatic actuators and the tip-in instability," J. Microelectromech. Syst., vol. 12 , pp. 656-671, 2003.

[21] P. B. Chu and K. S. J. Pister, "Analysis of closed-loop control of parallelplate electrostatic microgrippers," in Proc. IEEE Conf. Robot. Automat., San Diego, CA, May 1994, pp. 820-825.

[22] L. A. Rocha, E. Cretu, and R. F. Wolffenbuttel, "Displacement model for dynamic pull-in analysis and application in large-stroke actuators," in Proc. Eurosensors XVII, 2003, pp. 448-451.

[23] Y. Sun, D. Piyabongkarn, A. Sezen, B. J. Nelson, and R. Rajamani, "A high-aspect-ratio two axis electrostatic microactuator with extended travel range," Sens. Actuators A, Phys., vol. A 102, pp. 49-60, 2002.

[24] H. Troger and A. Steindl, Nonlinear Stability and Bifurcation Theory. New York: Springer-Verlag, 1991.

[25] T. Veijola, H. Kuisma, J. Lahdenpera, and T. Ryhanen, "Equivalent-circuit model of the squeezed gas film in a silicon accelerometer," Sens. Actuators A, Phys., vol. A 48, pp. 239-248, 1995.

[26] J. J. Blech, "On isothermal squeeze films," J. Lubrication Technol., vol. 105, pp. 615-620, 1983.

[27] T. Veijola, H. Kuisma, and J. Lahdenpera, "Compact large-displacement model for capacitive accelerometers," in Proc. MSM'99, San Juan, Puerto Rico, Apr. 19-21, 1999, pp. 218-221.

[28] S. Vemuri, G. K. Fedder, and T. Mukherjee, "Low-order squeeze film model for simulation of MEMS devices," in Proc. MSM'00, San Diego, CA, Mar. 27-29, 2000, pp. 205-208.

[29] T. Veijola, A. Pursula, and P. Raback, "Extending the validity of existing squeezed-film damper models with elongations of surface dimensions," in Proc. Nanotech2004, Boston, MA, Mar. 7-11, 2004, pp. 235-238.

[30] A. Medio and M. Lines, Nonlinear Dynamics. Cambridge, U.K.: Cambridge University Press, 2001.

[31] L. A. Rocha, E. Cretu, and R. F. Wolffenbuttel, "Analytical model for the pull-in time of low-Q MEMS devices," in Proc. Nanotech 2004, Boston, MA, USA, Mar. 7-11, 2004, pp. 271-274.

[32] K. Ogata, Modern Control Engineering, 3rd ed. Upper Saddle River, NJ: Prentice-Hall, 1997.

[33] . [Online]http://www.europractice.bosch.com/en/start/index.htm

[34] M. Offenberg, F. Lärmer, B. Elsner, H. Münzel, and W. Riethmüller, "Novel process for an integrated accelerometer," in Proc. Transducers 95 , vol. 1, 1995, pp. 589-593.

[35] M. Lemkin and B. E. Boser, "A three-axis micromachined accelerometer with a CMOS position-sense interface and digital offset-trim electronics," IEEE J. Solid-State Circuits, vol. 34, pp. 456-468, 1999.

[36] J. C. Lötters, W. Olthuis, P. H. Veltink, and P. Bergveld, "A sensitive differential capacitance to voltage converter for sensor applications," IEEE Trans. Instrum. Meas., vol. 48, pp. 89-96, 1999.

[37] MAX43x Wideband Transconductance Amplifier Family Datasheet. Sunnyvale, CA: Maxim Integrated Products.

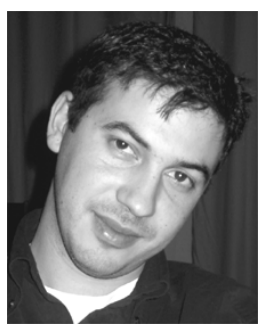

Luis Alexandre Rocha was born in Guimarães, Portugal, in 1977. He received the degree in electronic engineering from the University of Minho in 2000 and the Ph.D. degree from Delft University of Technology (cum laude) in 2005.

He was a Junior Engineer in Coelima during the year of 2000 and he has joined the MEMS group of ASML Netherlands, for six months in the beginning of 2005. Since July 2005, he has been at the Department of Industrial Electronics of the University of Minho, as a Postdoctoral Researcher in the field of wireless sensor networks and the study of nonlinear MEMS dynamic behavior. 


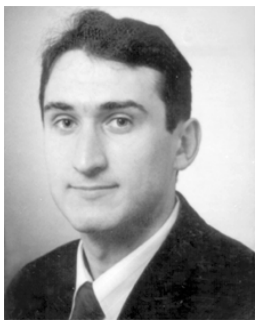

Edmond Cretu was born in Romania in 1965. He received the M.Sc. degree in electronic engineering from the Polytechnic University of Bucharest in 1989 and the Ph.D. degree from Delft University of Technology, The Netherlands, in 2003.

He was a Researcher at the Romanian Academy of Sciences and Associate Assistant at the Faculty of Electrical Engineering of the Polytechnic University of Bucharest. Since March 2000, he has been with Melexis Belgium, as a Senior Designer and Project Manager in the field of inertial systems, with emphasis on MEMS-based gyroscope systems.

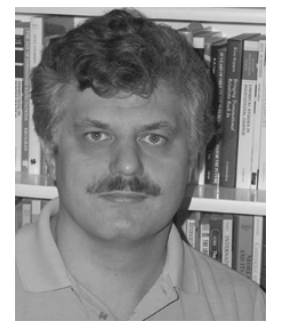

Reinoud F. Wolffenbuttel received the M.Sc. degree in 1984 and the Ph.D. degree in 1988, both from the Delft University of Technology, The Netherlands.

Between 1986 and 1993, he was an Assistant Professor and since 1993, he has been an Associate Professor at the Department of Microelectronics, Faculty of Information Technology and Systems of the Delft University of Technology and is involved in instrumentation and measurement in general and on-chip functional integration of microelectronic circuits and silicon sensor, fabrication compatibility issues, and micromachining in silicon and microsystems, in particular. He was a Visitor at the University of Michigan, Ann Arbor, in 1992, 1999, and 2001, Tohoku University, Sendai, Japan, in 1995 and EPFL Lausanne, Switzerland, in 1997.

Dr. Wolffenbuttel is the recipient of a 1997 NWO pioneer award. He served as General Chairman of the Dutch National Sensor Conference in 1996, Eurosensors in 1999, and the MicroMechanics Europe Workshop in 2003. 